\title{
Hypocretin and Nicotine Excite the Same Thalamocortical Synapses in Prefrontal Cortex: Correlation with Improved Attention in Rat
}

\author{
Evelyn K. Lambe, ${ }^{1}$ Peter Olausson, ${ }^{1}$ Nicole K. Horst, ${ }^{2}$ Jane R. Taylor, ${ }^{1}$ and George K. Aghajanian ${ }^{1,3}$ \\ ${ }^{1}$ Department of Psychiatry, ${ }^{2}$ Interdepartmental Neuroscience Program, and ${ }^{3}$ Department of Pharmacology, Yale University School of Medicine, New Haven, \\ Connecticut 06508
}

\begin{abstract}
Thalamic projections to prefrontal cortex are important for executive aspects of attention. Using two-photon imaging in prefrontal brain slices, we show that nicotine and the wakefulness neuropeptide hypocretin (orexin) excite the same identified synapses of the thalamocortical arousal pathway within the prefrontal cortex. Although it is known that attention can be improved when nicotine is infused directly into the midlayer of the prefrontal cortex in the rat, the effects of hypocretin on attention are not known. The overlap in thalamocortical synapses excited by hypocretin and nicotine and the lack of direct postsynaptic effects prompted us to compare their effects on a sustained and divided attention task in the rat. Similar to nicotine, infusions of hypocretin-2 peptide into the prefrontal cortex significantly improved accuracy under high attentional demand without effects on other performance measures. We show for the first time that hypocretin can improve attentional processes relevant to executive functions of the prefrontal cortex.
\end{abstract}

Key words: two-photon; orexin; divided attention; rat; $\alpha 4 \beta 2$ nicotinic acetylcholine receptors; behavior

\section{Introduction}

The executive attention network of the medial prefrontal cortex permits the sharing of attention among different potential sources of information (Dalley et al., 2004). This region of the prefrontal cortex receives projections from the midline and intralaminar nuclei of the thalamus (Berendse and Groenewegen, 1991), a region activated by attention-demanding tasks (Kinomura et al., 1996). Damage to part of this region is thought to result in concentration difficulties (Van der Werf et al., 1999). A recent large study (Van der Werf et al., 2003) found that the majority of patients with such thalamic lesions had deficits on tests of fronto-executive function and either simple or complex attention, although no one thalamic nucleus could be shown to be responsible for the attention deficits.

The thalamocortical synapse in the prefrontal cortex is the final synapse in the ascending arousal pathway. Neurotransmitters and drugs thought to stimulate this synapse have been hypothesized to improve executive aspects of attention (Pinault, 1995). For example, many reports suggest that nicotine can enhance attention (for review, see Rezvani and Levin, 2001). Such an effect is congruent with recent indirect evidence that levels of

Received Feb. 22, 2005; revised April 19, 2005; accepted April 20, 2005.

This work was supported by a Kevin Hines National Alliance for Research on Schizophrenia and Depression Young Investigator Award (E.K.L.), by Yale University Transdisciplinary Tobacco Use Research Center Pilot Grant 1 P50 AA15632 (E.K.L.), and by National Institutes of Health Grants MH17871 (G.K.A.) and DA11717 (J.R.T.). We thank Drs. Meenakshi Alreja, Daniel McGehee, and Marina Picciotto for valuable discussions. Elin Lof, Nancy Margiotta, and Damaris Sheffield gave important technical assistance.

Correspondence should be addressed to Dr. Evelyn K. Lambe, Ribicoff Research, Room 307, Connecticut Mental Health Center, 34 Park Street, New Haven, CT 06508. E-mail: evelyn.lambe@yale.edu.

DOI:10.1523/JNEUROSCI.0719-05.2005

Copyright $\odot 2005$ Society for Neuroscience $\quad$ 0270-6474/05/255225-05\$15.00/0 nicotine similar to peak blood levels in human smokers can excite a population of thalamic terminals through high-affinity $\alpha 4 \beta 2$ nicotinic acetylcholine receptors in the absence of direct postsynaptic effects (Lambe et al., 2003). The loss of thalamic terminals after lesion prevents this effect, consistent with reductions in high-affinity nicotinic binding after thalamic lesions (Gioanni et al., 1999). Direct infusion of nicotine into the prefrontal cortex in the rat can enhance divided attention to a degree similar to that of systemic nicotine (Hahn et al., 2003). The improvement of attention with nicotine is unusual in that it persists despite chronic use (Hahn and Stolerman, 2002) and may be underappreciated as a reason that people, particularly those who have attentional problems attributable to an underlying psychiatric illness such as schizophrenia, are much more likely to smoke.

Much anecdotal evidence suggests that people with narcolepsy, who lack hypocretin, the neuropeptide critical for wakefulness, have problems concentrating. This has typically been suggested to result solely from sleepiness. However, a recent study that controlled for wakefulness found that individuals with narcolepsy have selective deficits in flexible or divided attention (Rieger et al., 2003). The attention-enhancing effects of nicotine may explain why some people with narcolepsy perceive cigarette smoking to be helpful. The mechanism by which nicotine in the prefrontal cortex can improve attention is not known. Although hypocretin peptides have been shown recently to excite thalamocortical synapses in the prefrontal cortex (Lambe and Aghajanian, 2003), to date, there is no direct evidence for a role of hypocretin in attention.

Using two-photon imaging at single identified thalamocortical synapses in the rat prefrontal slice, we can visualize and compare activated synapses. Here, we demonstrate that nicotine and 
hypocretin-2 peptide excite overlapping thalamocortical pathways in the prefrontal cortex. Because stimulation of the final synapse in the ascending arousal pathway would have behavioral consequences on tasks of prefrontal function, we also show that infusions of nicotine or hypocretin into the rat prefrontal cortex improve attentional function.

\section{Materials and Methods}

Electrophysiology. Prefrontal cortical slices $(400 \mu \mathrm{m})$ were prepared from Sprague Dawley rats $(80-250$ g) as described previously (Lambe et al., 2003) and in accordance with the policies of the Yale University Institutional Animal Care and Use Committee. Except where mentioned, drugs and exogenous neurotransmitters were applied in the rapidly flowing $(\sim 3 \mathrm{ml} / \mathrm{min})$ bath. A modified U-tube (Y-tube) was used for rapid application of nicotine and acetylcholine (Wu et al., 2003). SSR591813 $[(5 a S, 8 S, 10 a R)-5 a, 6,9,10$-tetrahydro, $7 \mathrm{H}, 11 \mathrm{H}$-8,10a-methanopyrido $\left[2^{\prime}, 3^{\prime}: 5,6\right]$ pyrano[2,3-d]azepine] was a gift from Sanofi-Synthélabo (Montpellier, France). Hypocretin-2 peptide was a gift from Dr. Anthony van den Pol (Yale University) and was used because it preferentially stimulates hypocretin receptor 2 and not hypocretin receptor 1 at the concentrations used.

Thalamocortical terminal labeling and two-photon imaging. Thalamocortical synapses were labeled and imaged as described previously (Lambe and Aghajanian, 2003). In brief, thalamocortical axons were labeled with in vivo electroporation of Phaseolus vulgaris conjugated to Alexa 488 (Molecular Probes, Eugene, OR). After 3-4 d to allow anterograde transport to the prefrontal cortex, prefrontal cortical slices were obtained. Layer V prefrontal pyramidal neurons were labeled through the patch pipette with a "green" calcium indicator, Oregon Green BAPTA-1 (100-200 $\mu \mathrm{M})$, and a "red" reference dye, Alexa 594 (20-30 $\mu \mathrm{M})$. This combination renders the dendrites and spines "orange" in appearance and thus easily distinguishable from the green thalamocortical axons. Low-magnesium artificial CSF (ACSF) $\left(0.25 \mathrm{~mm} \mathrm{Mg}^{2+}\right)$ was used to enhance NMDA-mediated calcium currents. To minimize photobleaching, scans were taken every $2 \mathrm{~s}$ during baseline, bath application of nicotine or hypocretin, and washout. The order of drug and control scans was varied. Spines showing a change with hypocretin or nicotine at least four SDs from baseline $(Z \geq 4)$ were classified as "responders." Spontaneous EPSCs (sEPSCs)were recorded throughout imaging, and lengthy washout periods were allowed between drug applications. A brief depolarization-induced burst was used as a control to show that it was possible to detect a calcium increase in spines that did not appear to respond to hypocretin or nicotine.

Behavior. Ten adult male Sprague Dawley rats were trained and tested in a self-paced task of visuospatial sustained and divided attention (Jentsch and Taylor, 2003). In this task, food-restricted animals are trained to respond to a visual target stimulus that is randomly displayed on either the left or right side after completion of a sustained response in the center nose-poke aperture. Successful detection and response in the target stimulus aperture were reinforced with a food pellet. Chamber lights were extinguished for $3 \mathrm{~s}$ if the response was incorrect, if no response was made, or if the animal responded before the presentation of the target stimulus. The session was terminated after initiation of 160 trials or after $1 \mathrm{~h}$. Stimulus-duration times were variable $(0.3,0.5,0.7$, or $1.0 \mathrm{~s}$ ) and randomly presented within each session. Thus, effects of the present treatments could be evaluated under variable attentional demand. The measures obtained included information about correct responses, error types, reaction times, and initiation latencies.

After acquisition of the attention task, all animals were implanted with bilateral stainless-steel cannulas aimed at the medial prefrontal cortex (anteroposterior, $+3.2 \mathrm{~mm}$; mediolateral, $\pm 0.5 \mathrm{~mm}$; and dorsoventral, $-3.0 \mathrm{~mm}$ ). After recovery, rats were retrained to criterion on the attention task and habituated to infusion procedure. The experiment was subsequently performed according to a Latin square design. Here, all animals received infusions $(1.0 \mu \mathrm{l}$ at $0.25 \mu \mathrm{l} / \mathrm{min}$; syringes were left in place for 2 min after infusion to allow for diffusion) of sterile saline, nicotine, or either of the two doses of hypocretin-2 peptide immediately
A

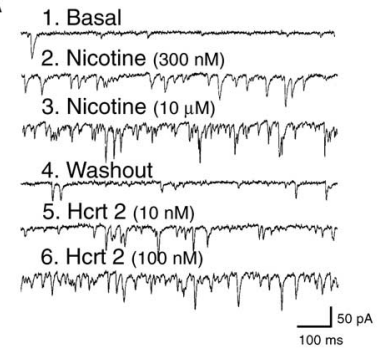

B

C

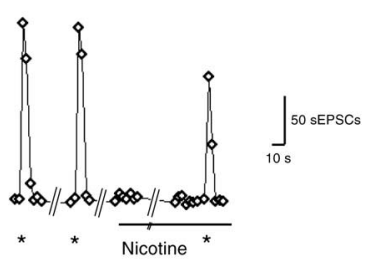

D

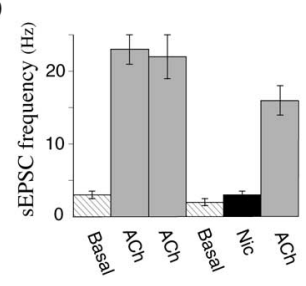

Figure 1. Electrophysiological effects of nicotine and hypocretin on sEPSCs in layer V pyramidal neurons of prefrontal cortex. $A$, Sweeps in voltage clamp showing SEPSCS under basal conditions (1), in the presence of two concentrations of nicotine [300 nм (2) and $10 \mu \mathrm{m}(3)$ ], after a 30 min washout (4), and in the presence of two concentrations of hypocretin- 2 peptide [10 nм (5) and $100 \mathrm{~nm}(6)] . \boldsymbol{B}$, Bar graph showing sEPSC frequency for hypocretin-2 peptide and nicotine in varied order (mean \pm SEM; $n=5$ neurons), as well as SSR591813, a partial agonist of $\alpha 4 \beta 2$ nicotinic receptors ( $n=5$ neurons). C, The graph shows sEPSCs (in 10 s bins) from one pyramidal neuron in the presence of $0.5 \mu \mathrm{m}$ atropine to block muscarinic receptors. Asterisks denote Y-tube application of acetylcholine (1 $\mathrm{mm} ; 5 \mathrm{~s})$. There was a 5 min washout period between the first and second Y-tube applications. Nicotine $(50 \mathrm{~nm})$ was then applied in the bath for $15 \mathrm{~min}$ before a final $Y$-tube application of acetylcholine. $D$, The bar graph shows sEPSC frequency (mean \pm SEM; $n=5$ neurons) elicited by $Y$-tube applications of acetylcholine under these conditions. Error bars represent SEM. Hcrt, Hypocretin; Nic, nicotine.

before behavioral testing. Only animals with confirmed infusion placements were included in the statistical analyses.

\section{Results}

\section{Electrophysiology}

Levels of nicotine similar to peak blood levels in human smokers $(\sim 300 \mathrm{nM})$ elicit an increase in glutamate release onto layer $\mathrm{V}$ pyramidal neurons of the prefrontal cortex of the rat (Lambe et al., 2003). This increase, measured by an increase in sEPSCs, has been suggested to result from excitation of presynaptic highaffinity $\alpha 4 \beta 2$ nicotinic acetylcholine receptors on thalamocortical terminals, and it is not seen in the prefrontal slice of $\beta 2$ subunit knock-out mice (Lambe et al., 2003). As with nicotine, stimulation of hypocretin receptor 2 excites thalamocortical synapses without directly affecting postsynaptic layer $\mathrm{V}$ cells in the prefrontal cortex (Lambe and Aghajanian, 2003). Therefore, we compared sEPSCs elicited by nicotine and hypocretin-2 peptide in layer $\mathrm{V}$ pyramidal neurons in the brain slice of the rat medial prefrontal cortex. Bath application of either nicotine or hypocretin induces a prolonged increase in sEPSCs (Fig. 1A). A novel selective $\alpha 4 \beta 2$ partial agonist, SSR591813 (Cohen et al., 2003), was compared with nicotine to assess its ability to elicit glutamate release from thalamocortical synapses, as shown in Figure $1 B$.

Because $\alpha 4 \beta 2$ nicotinic acetylcholine receptors in some brain regions are vulnerable to desensitization (Alkondon et al., 2000; Mansvelder et al., 2002; Wooltorton et al., 2003), we also used a Y-tube (Wu et al., 2003) to allow rapid local application of nicotine. The increase in sEPSCs with either nicotine or acetylcholine (in the presence of $0.5 \mu \mathrm{M}$ atropine to block muscarinic receptors) was found to be more rapid (latency of $\sim 500 \mathrm{~ms}$ ) than bath application (latency of $\sim 3 \mathrm{~s}$ ). However, the peak increase in 
sEPSCs was marginally less than that with rapid bath application, consistent with more localized application affecting fewer thalamocortical terminals. We assessed desensitization over a longer time course by combining bath application of nicotine with Y-tube acetylcholine ( $\mathrm{ACh}$ ) challenges in the presence of 0.5 $\mu \mathrm{M}$ atropine. These increases in sEPSCs could be almost completely suppressed by application of dihydro- $\beta$-erythroidine $(\mathrm{DH} \beta \mathrm{E})(10 \mu \mathrm{M}$; mean $\pm \mathrm{SEM},-88 \pm 3 \% ; n=4)$, consistent with mediation by $\alpha 4 \beta 2$ nicotinic receptors. Unexpectedly, sEPSCs induced by Y-tube ACh application were markedly preserved after prolonged exposure to a low level of nicotine (50 nM; 15 min; $n=5$ ) (Fig. 1C,D). Greater, but still not complete, desensitization could be seen with prolonged application of a much higher level of nicotine ( $300 \mathrm{~nm} ; n=2$; data not shown).

Hypocretin-elicited sEPSCs were not altered by previous application of nicotine $(22.3 \pm 1.5$ vs $20.1 \pm 1.8 \mathrm{~Hz}$ peak sEPSC frequency; $n=3$ ), the $\alpha 4 \beta 2$ nicotinic antagonist $\mathrm{DH} \beta \mathrm{E}(10 \mu \mathrm{M}$; $20.9 \pm 2.0$ vs $21.5 \pm 2.6 \mathrm{~Hz}$ peak sEPSC frequency; $n=3)$, or the muscarinic antagonist atropine $(0.5 \mu \mathrm{M} ; 18.5 \pm 1.8$ vs $17.6 \pm 1.3$ $\mathrm{Hz}$ peak sEPSC frequency; $n=2)$. Neither nicotine nor hypocretin directly depolarized layer II/III $(n=9)$ or layer V pyramidal neurons $(n=27)$.

\section{Two-photon imaging}

A recently developed triple-labeling technique permits the examination of neurotransmitter-induced synaptic activity at single identified synapses (Lambe and Aghajanian, 2003). We used this technique to compare the excitation of thalamocortical synapses by nicotine and hypocretin. Ultrastructural work has shown that layer $\mathrm{V}$ pyramidal neurons in the prefrontal cortex receive thalamocortical synapses (Marini et al., 1996). Spines appearing to be in apposition to Phaseolus-labeled thalamic boutons or axons were compared with spines in focus in the same field but not in apposition to a labeled terminal. In six imaging fields $(n=37$ spines in focus), all spines with apparent appositions to a labeled thalamocortical bouton responded to both nicotine and hypocretin (Fig. 2) ( $n=6$ spines) with large, repeated calcium transients. These calcium transients could be eliminated with the application of $2 \mathrm{mM} \mathrm{Mg}^{2+} \mathrm{ACSF}$ or $50 \mu \mathrm{M} \mathrm{APV} \mathrm{(data} \mathrm{not} \mathrm{shown),}$ congruent with their mediation by postsynaptic NMDA receptors (Lambe and Aghajanian, 2003). Spontaneous EPSCs were recorded throughout imaging and increased significantly during application of nicotine and hypocretin (data not shown). The order of application had no apparent effect on the frequency or magnitude of the response. As shown in Figure 2, there were no spontaneous large calcium transients at these spines during control scans. Of 31 nearby spines, which were in focus but did not have apparent appositions with thalamocortical terminals, only three responded to application of hypocretin or nicotine, whereas a brief burst of spikes elicited transients at all spines. Of the three spines that responded to hypocretin or nicotine, despite lacking apparent thalamic appositions, two responded to both nicotine and hypocretin, and one responded to hypocretin only. In summary, $100 \%$ of spines with apparent thalamocortical appositions responded to application of both nicotine and hypocretin, whereas $<10 \%$ of spines lacking appositions responded to either nicotine or hypocretin.

\section{Behavior}

To compare the effects of hypocretin and nicotine on sustained and divided attention, we used a modified version of the rat analog of the human continuous-performance task. Here, rats were trained to perform on a self-paced divided-attention task in
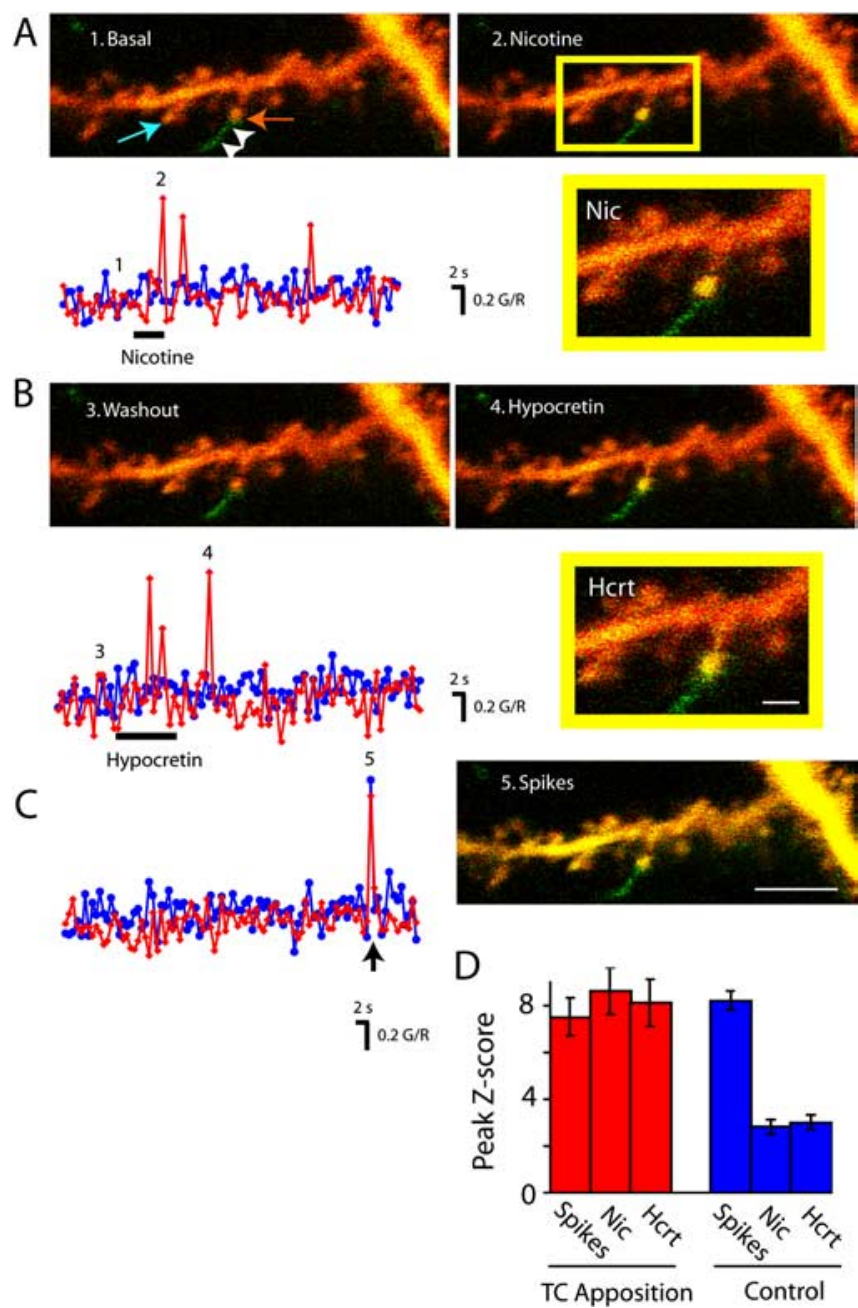

Figure 2. Postsynaptic calcium transients elicited by nicotine and hypocretin at a single spine in apposition with a thalamocortical terminal. $\boldsymbol{A}$, Images show spines on a tertiary dendritic branch of a layer V pyramidal neuron (1) under baseline conditions and (2) during a nicotine-elicited $(10 \mu \mathrm{m})$ postsynaptic calcium transient at the spine in apparent apposition to a thalamocortical terminal (inset). Red and blue graph lines show the green/red (G/R) intensity ratio obtained from regions of interest on the spines, indicated by the red and blue arrows, respectively. $\boldsymbol{B}$, Images show spines (3) 30 min after nicotine washout and (4) during a hypocretin-elicited (100 nm) postsynaptic calcium transient. The graph shows hypocretinelicited changes in $\mathrm{G} / \mathrm{R}$ intensity ratio at the same spines as above. Scale bar, $2 \mu \mathrm{m}$. C, Image showing (5) high calcium elicited by a burst of action potentials. Scale bar, $5 \mu \mathrm{m}$. The graph shows control series of images acquired without application of neurotransmitter or drug. The arrow indicates a brief depolarizing current injection used to elicit the burst of spikes. $\boldsymbol{D}$, The bar graph at the bottom right shows peak Z-scores (mean \pm SEM) for G/R ratio in spines with ( $n=$ 6 spines) and without ( $n=31$ spines) apparent thalamocortical (TC) appositions in response to nicotine, hypocretin, and the burst of spikes. Error bars represent SEM. Nic, Nicotine; Hcrt, hypocretin.

which animals have to monitor target stimuli presented at one of two spatial locations and respond appropriately to that location [modified from the study by Jentsch and Taylor (2003)]. In this study, we infused saline, hypocretin (100 or $300 \mathrm{pmol}$ ), or nicotine $(17 \mathrm{nmol})$ bilaterally into the medial prefrontal cortex immediately before testing according to a Latin square design. The dose of nicotine chosen for comparison with hypocretin was based on its effect in a previous study (Hahn et al., 2003). The attentional demand was randomly varied within the test session using variable target-stimulus duration times between $0.3 \mathrm{~s}$ (most difficult, $<55 \%$ accuracy with saline infusion) and $1.0 \mathrm{~s}$ (least difficult, $75 \%$ accuracy with saline infusion). All rats $(n=10)$ 
A

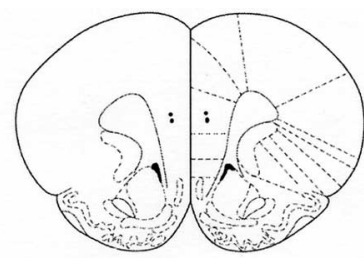

Bregma $2.70 \mathrm{~mm}$

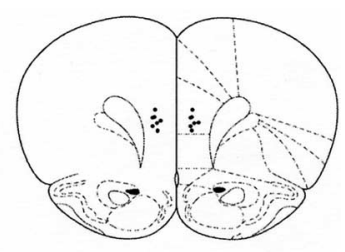

Bregma $3.20 \mathrm{~mm}$
B

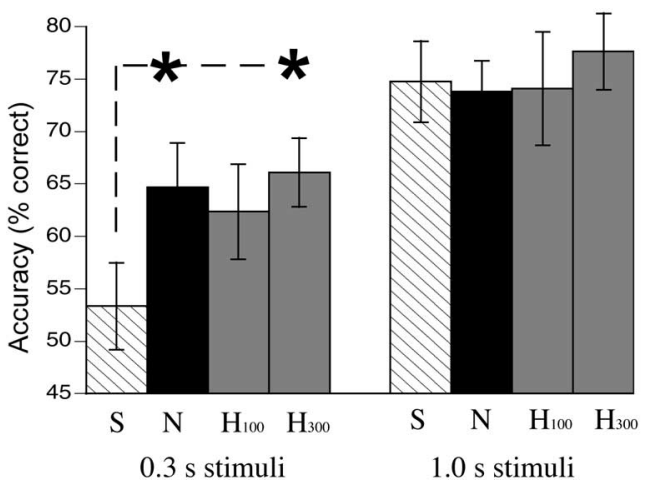

Figure 3. Behavioral effects of bilateral infusions of nicotine or hypocretin into the medial prefrontal cortex on a task of sustained, divided attention. $A$, Schematic showing midlayer infusion sites in the medial prefrontal cortex for the nine rats included in the statistical analysis. $\boldsymbol{B}$, Performance accuracy (mean \pm SEM; $n=9$ rats) at the 0.3 and 1.0 s stimulus durations after bilateral infusions of saline (S), two doses of hypocretin-2 peptide ( $\mathrm{H} ; 100$ and $300 \mathrm{pmol})$, and nicotine $(\mathrm{N} ; 17 \mathrm{nmol})$ into the medial prefrontal cortex. The effect of hypocretin was dose related. Nicotine and the higher dose of hypocretin $(300 \mathrm{pmol})$ each significantly increased accuracy at the most challenging stimulus interval $\left({ }^{*} p<0.01\right)$. Error bars represent SEM.

received all treatments, including both doses of hypocretin, on consecutive days with a $24 \mathrm{~h}$ washout between tests. After this series of experiments, the animals were killed, and the infusion site was verified histologically. Only animals with cannula placements in the midlayer of the medial prefrontal cortex $(n=9)$, as shown in Figure $3 A$, were included in the statistical analysis. The relatively large infusion volume may allow diffusion to several prefrontal cortical areas.

Our a priori hypothesis that nicotine and hypocretin would improve attentional performance under demanding conditions was tested using ANOVA with treatment and stimulus duration $(1.0 \mathrm{~s}$, low attentional demand; $0.3 \mathrm{~s}$, high attentional demand) as repeated factors. This analysis demonstrated a significant treatment by stimulus duration interaction $\left(F_{(3,24)}=3.872 ; p \leq 0.05\right)$. Post hoc analysis using Dunnett's $t$ test showed that both nicotine and hypocretin (300 pmol dose) improved accuracy only when the attention task was most demanding $(0.3 \mathrm{~s}$ stimulus duration, $p<0.01$, both treatments; $1.0 \mathrm{~s}$ stimulus duration, not significant), as shown in Figure 3B. No difference was seen in reaction time, response inhibition, trial initiation latency, or the number of trials completed (see supplemental results, available at www. jneurosci.org as supplemental material). Interestingly, the rat that was excluded from the analysis because of incorrect cannula placement (orbital instead of medial prefrontal cortex) did not show improvement of attentional performance with hypocretin or nicotine.

\section{Discussion}

We show that nicotine and the wakefulness peptide hypocretin excite the same single identified thalamocortical synapses in pre- frontal cortical slices in the absence of direct postsynaptic effects on layer II/III or layer V pyramidal neurons. Furthermore, nicotine or hypocretin infused into the prefrontal cortex similarly improves performance on a task of sustained and divided attention, specifically at the most demanding, shortest target duration. This is the first study to demonstrate a role for hypocretin in attention and to suggest a mechanism underlying the similar ability of nicotine and hypocretin to enhance performance under conditions of high attentional demand.

The midline and intralaminar nuclei of the thalamus project to the prefrontal cortex (Berendse and Groenewegen, 1991) and have long been implicated in attention. Early electrophysiological work showed that neurons in these nuclei promote cortical activation (Dempsey and Morison, 1942). Interestingly, thalamic axons are susceptible to the generation of terminal spikes (for review, see Pinault, 1995) that can be triggered by neurotransmitters. The generation of such spikes in thalamocortical terminals by hypocretin or nicotine would explain the TTX sensitivity of their effects in the prefrontal cortex (Gioanni et al., 1999; Lambe and Aghajanian, 2003; Lambe et al., 2003). In vivo, these terminal spikes would propagate antidromically back to the thalamus, in which they have been hypothesized to coordinate burst firing (Pinault, 1995). With the exception of subgriseal neurons (Bayer et al., 2004), very few neurons in the prefrontal cortex respond directly to hypocretin (van den Pol et al., 1998).

The medial prefrontal cortex is unusual in that it is a discrete projection target of almost one-third of hypocretin neurons (Fadel et al., 2002). It is not known whether release from these terminals varies with behavioral state. The release of hypocretin in other brain areas has been shown to be highest during waking (Yoshida et al., 2001; Kiyashchenko et al., 2002). However, studies using microdialysis lack the time resolution to assess hypocretin fluctuations during complex behaviors, such as the performance of an attention task. It would be interesting to determine whether prefrontally projecting neurons in this region vary their firing rate with attentional demand. One interesting difference between hypocretin and nicotine is that hypocretin would excite thalamocortical terminals predominantly in the prefrontal cortex (Lambe and Aghajanian, 2003), whereas nicotine could excite thalamocortical terminals throughout the cortex. The effects of exciting thalamocortical terminals in other regions of the cortex warrant additional study.

A role for prefrontal hypocretin in aspects of attention may explain why people with narcolepsy, who lack hypocretin, report concentration difficulties. Although such difficulties have long been attributed to sleepiness, a recent study that controlled for alertness found selective deficits in divided and flexible attention in people with narcolepsy (Rieger et al., 2003). An attentional benefit from nicotine may make cigarette smoking more appealing to people with concentration difficulties, despite its evident dangers.

The similarity between the rats' improvement on the most challenging aspect of the attention task with hypocretin and with nicotine is striking in part because of the recent debate about whether high-affinity $\alpha 4 \beta 2$ nicotinic receptors are activated or desensitized during smoking. The effect of nicotine on divided attention is unusual in that it occurs with both acute and chronic exposure to nicotine (Hahn and Stolerman, 2002) and appears to be mediated by high-affinity $\alpha 4 \beta 2$ nicotinic receptors (Blondel et al., 2000; Grottick and Higgins, 2000). In some studies, $\alpha 4 \beta 2$ nicotinic receptors on interneurons have been shown to be almost completely desensitized by low levels of nicotine (Wooltorton et al., 2003) [but see Alkondon et al. (2000) and Mansvelder et 
al. (2002), which required higher concentrations of nicotine to achieve a similar desensitization]. In the present study, exposure to a low level of nicotine only partially suppressed acetylcholineelicited sEPSCs (in the presence of atropine), suggesting that the $\alpha 4 \beta 2$ nicotinic receptors on thalamocortical terminals may be more resistant to desensitization. Our findings are congruent with an in vivo microdialysis study showing that prolonged locally administered nicotine increases prefrontal glutamate in a TTX-sensitive manner that can be blocked by local application of the $\alpha 4 \beta 2$ nicotinic antagonist $\mathrm{DH} \beta \mathrm{E}$ (Gioanni et al., 1999). It is possible that the stoichiometry or local environment of highaffinity nicotinic receptors on thalamocortical projections results in less rapid desensitization than that observed for $\alpha 4 \beta 2$ receptors on interneurons. Such resistance to desensitization would be consistent with the persistent ability of nicotine to improve attention in humans and rodents.

Thalamocortical terminals in the prefrontal cortex are the final synapse in the ascending arousal pathway. The presence of a neurotransmitter, such as hypocretin, or a drug, such as nicotine, that elicits terminal spiking in thalamocortical projections may promote midline and intralaminar neurons to enter bursting mode. Thalamic bursts have been shown to potently activate the cerebral cortex (Swadlow and Gusev, 2001), which may augment the alerting response to faint stimuli and permit more accurate allocation of limited attentional resources. The ability of nicotine and hypocretin to mimic each other in the prefrontal cortex by exciting thalamocortical terminals and enhancing divided attention may yield insight into potential novel treatments for psychiatric and neurological disorders.

\section{References}

Alkondon M, Pereira EF, Almeida LE, Randall WR, Albuquerque EX (2000) Nicotine at concentrations found in cigarette smokers activates and desensitizes nicotinic acetylcholine receptors in CA1 interneurons of rat hippocampus. Neuropharmacology 39:2726-2739.

Bayer L, Serafin M, Eggermann E, Saint-Mleux B, Machard D, Jones BE, Muhlethaler M (2004) Exclusive postsynaptic action of hypocretinorexin on sublayer 6b cortical neurons. J Neurosci 24:6760-6764.

Berendse HW, Groenewegen HJ (1991) Restricted cortical termination fields of the midline and intralaminar thalamic nuclei in the rat. Neuroscience 42:73-102.

Blondel A, Sanger DJ, Moser PC (2000) Characterisation of the effects of nicotine in the five-choice serial reaction time task in rats: antagonist studies. Psychopharmacology (Berl) 149:293-305.

Cohen C, Bergis OE, Galli F, Lochead AW, Jegham S, Biton B, Leonardon J, Avenet P, Sgard F, Besnard F, Graham D, Coste A, Oblin A, Curet O, Voltz C, Gardes A, Caille D, Perrault G, George P, Soubrie P, et al. (2003) SSR591813, a novel selective and partial alpha4beta2 nicotinic receptor agonist with potential as an aid to smoking cessation. J Pharmacol Exp Ther 306:407-420.

Dalley JW, Cardinal RN, Robbins TW (2004) Prefrontal executive and cognitive functions in rodents: neural and neurochemical substrates. Neurosci Biobehav Rev 28:771-784.

Dempsey EW, Morison RS (1942) The production of rhythmically recurrent cortical potentials after localized thalamic stimulation. Am J Physiol 135:293-300.

Fadel J, Bubser M, Deutch AY (2002) Differential activation of orexin neurons by antipsychotic drugs associated with weight gain. J Neurosci 22:6742-6746.
Gioanni Y, Rougeot C, Clarke PB, Lepouse C, Thierry AM, Vidal C (1999) Nicotinic receptors in the rat prefrontal cortex: increase in glutamate release and facilitation of mediodorsal thalamo-cortical transmission. Eur J Neurosci 11:18-30.

Grottick AJ, Higgins GA (2000) Effect of subtype selective nicotinic compounds on attention as assessed by the five-choice serial reaction time task. Behav Brain Res 117:197-208.

Hahn B, Stolerman IP (2002) Nicotine-induced attentional enhancement in rats: effects of chronic exposure to nicotine. Neuropsychopharmacology 27:712-722.

Hahn B, Shoaib M, Stolerman IP (2003) Involvement of the prefrontal cortex but not the dorsal hippocampus in the attention-enhancing effects of nicotine in rats. Psychopharmacology (Berl) 168:271-279.

Jentsch JD, Taylor JR (2003) Sex-related differences in spatial divided attention and motor impulsivity in rats. Behav Neurosci 117:76-83.

Kinomura S, Larsson J, Gulyas B, Roland PE (1996) Activation by attention of the human reticular formation and thalamic intralaminar nuclei. Science 271:512-515.

Kiyashchenko LI, Mileykovskiy BY, Maidment N, Lam HA, Wu MF, John J, Peever J, Siegel JM (2002) Release of hypocretin (orexin) during waking and sleep states. J Neurosci 22:5282-5286.

Lambe EK, Aghajanian GK (2003) Hypocretin (orexin) induces calcium transients in single spines postsynaptic to identified thalamocortical boutons in prefrontal slice. Neuron 40:139-150.

Lambe EK, Picciotto MR, Aghajanian GK (2003) Nicotine induces glutamate release from thalamocortical terminals in prefrontal cortex. Neuropsychopharmacology 28:216-225.

Mansvelder HD, Keath JR, McGehee DS (2002) Synaptic mechanisms underlie nicotine-induced excitability of brain reward areas. Neuron 33:905-919.

Marini G, Pianca L, Tredici G (1996) Thalamocortical projection from the parafascicular nucleus to layer $\mathrm{v}$ pyramidal cells in frontal and cingulate areas of the rat. Neurosci Lett 203:81-84.

Pinault D (1995) Backpropagation of action potentials generated at ectopic axonal loci: hypothesis that axon terminals integrate local environmental signals. Brain Res Brain Res Rev 21:42-92.

Rezvani AH, Levin ED (2001) Cognitive effects of nicotine. Biol Psychiatry 49:258-267.

Rieger M, Mayer G, Gauggel S (2003) Attention deficits in patients with narcolepsy. Sleep 26:36-43.

Swadlow HA, Gusev AG (2001) The impact of "bursting" thalamic impulses at a neocortical synapse. Nat Neurosci 4:402-408.

van den Pol AN, Gao XB, Obrietan K, Kilduff TS, Belousov AB (1998) Presynaptic and postsynaptic actions and modulation of neuroendocrine neurons by a new hypothalamic peptide, hypocretin/orexin. J Neurosci 18:7962-7971.

Van der Werf YD, Weerts JG, Jolles J, Witter MP, Lindeboom J, Scheltens P (1999) Neuropsychological correlates of a right unilateral lacunar thalamic infarction. J Neurol Neurosurg Psychiatry 66:36-42.

Van der Werf YD, Scheltens P, Lindeboom J, Witter MP, Uylings HB, Jolles J (2003) Deficits of memory, executive functioning and attention following infarction in the thalamus; a study of 22 cases with localised lesions. Neuropsychologia 41:1330-1344.

Wooltorton JR, Pidoplichko VI, Broide RS, Dani JA (2003) Differential desensitization and distribution of nicotinic acetylcholine receptor subtypes in midbrain dopamine areas. J Neurosci 23:3176-3185.

Wu M, Hajszan T, Leranth C, Alreja M (2003) Nicotine recruits a local glutamatergic circuit to excite septohippocampal GABAergic neurons. Eur J Neurosci 18:1155-1168.

Yoshida Y, Fujiki N, Nakajima T, Ripley B, Matsumura H, Yoneda H, Mignot E, Nishino S (2001) Fluctuation of extracellular hypocretin-1 (orexin A) levels in the rat in relation to the light-dark cycle and sleep-wake activities. Eur J Neurosci 14:1075-1081. 